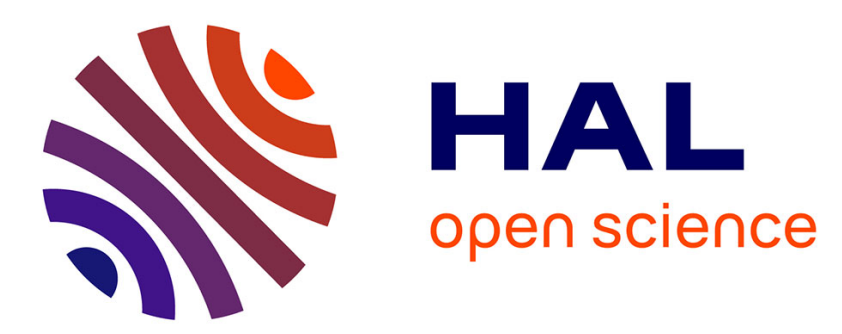

\title{
Design of personalized wearable haptic interfaces to account for fingertip size and shape
}

Monica Malvezzi, Francesco Chinello, Domenico Prattichizzo, Claudio Pacchierotti

\section{- To cite this version:}

Monica Malvezzi, Francesco Chinello, Domenico Prattichizzo, Claudio Pacchierotti. Design of personalized wearable haptic interfaces to account for fingertip size and shape. IEEE Transactions on Haptics (ToH), 2021, 14 (2), pp.266 - 272. 10.1109/TOH.2021.3076106 . hal-03207560

\section{HAL Id: hal-03207560 \\ https://hal.inria.fr/hal-03207560}

Submitted on 25 Apr 2021

HAL is a multi-disciplinary open access archive for the deposit and dissemination of scientific research documents, whether they are published or not. The documents may come from teaching and research institutions in France or abroad, or from public or private research centers.
L'archive ouverte pluridisciplinaire HAL, est destinée au dépôt et à la diffusion de documents scientifiques de niveau recherche, publiés ou non, émanant des établissements d'enseignement et de recherche français ou étrangers, des laboratoires publics ou privés. 


\title{
Design of personalized wearable haptic interfaces to account for fingertip size and shape
}

\author{
Monica Malvezzi ${ }^{1, *}$, Francesco Chinello ${ }^{3, *}$, Domenico Prattichizzo ${ }^{1,2, \ddagger}$, Claudio Pacchierotti ${ }^{4, \ddagger}$
}

\begin{abstract}
The size and shape of fingertips vary significantly across humans, making it challenging to design wearable fingertip interfaces suitable for everyone. Although deemed important, this issue has often been neglected due to the difficulty of customizing devices for each different user. This paper presents an innovative approach for automatically adapting the hardware design of a wearable haptic interface for a given user. We consider a 3-DoF fingertip cutaneous device, composed of a static body and a mobile platform linked by three articulated legs. The mobile platform is capable of making and breaking contact with the finger pulp and re-angle to replicate contacts with arbitrarily-oriented surfaces. We analyze the performance of this device as a function of its main geometrical dimensions. Then, starting from the user's fingertip characteristics, we define a numerical procedure that best adapts the dimension of the device to (i) maximize the range of renderable haptic stimuli, (ii) avoid unwanted contacts between the device and the skin, (iii) avoid singular configurations, and (iv) minimize the device encumbrance and weight. Together with the mechanical analysis and evaluation of the adapted design, we present a MATLAB script that calculates the device dimensions customized for a target fingertip as well as an online CAD utility for generating a ready-to-print STL file of the personalized design.
\end{abstract}

\section{INTRODUCTION}

Wearable haptic interfaces for the fingertips are growing interest in the field of haptics [1]. All these devices have been designed for users with certain fingertip characteristics, without considering the large differences in fingertip's size and shape across humans [2], [3]. These physical differences can significantly distort the perception of haptic feedback provided by fingertip haptic devices (see Fig. 1). Although this problem has been widely acknowledged [1], [4], little work has been done to address it and no easy-to-use or automatic technique is available to adapt the design of a wearable device for a target user. One example has been presented by Young et al. [5], where the rendering algorithm of a fingertip device is adapted to account for the fingertip size and shape. However, no hardware/design adjustment is considered.

This paper introduces an automatic technique to personalize the structure and dimensions of a wearable fingertip device for a given fingertip. As a representative example, we consider the popular wearable fingertip device of Chinello et al. [6], shown in Fig. 2. Starting from the geometrical characteristics of the user's fingertip, we define a numerical procedure that automatically adapts the dimensions of the static body, mobile platform, and articulated legs to $i$ ) reach all the parts of the fingertip needed for the given interaction, ii) avoid undesired contacts between the device and the finger (e.g., lateral contacts), iii) avoid kinematic singularities in all the

\footnotetext{
${ }^{1}$ Dept. Information Eng. and Mathematics, Univ. Siena - Siena, Italy.

${ }^{2}$ Dept. Advanced Robotics, Istit. Italiano di Tecnologia - Genova, Italy.

${ }^{3}$ Dept. Business Dev. and Tech., Aarhus Univ. - Herning, Denmark.

${ }^{4}$ CNRS, Univ Rennes, Inria, IRISA - Rennes, France.

$*, \ddagger$ These two couples of authors contributed equally to this work.
}

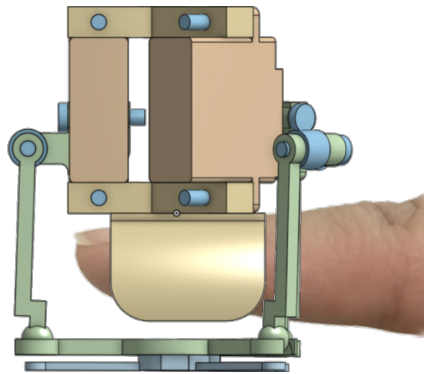

(a) Small fingertip wearing the device.

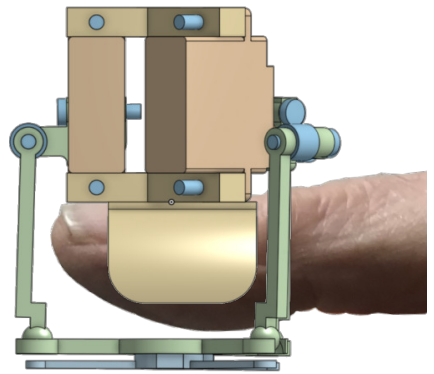

(b) Large fingertip wearing the device.
Fig. 1. Problem: the same fingertip haptic device will elicit different sensations on fingertips having different size and shape. Our personalization approach optimizes the device design for a target fingertip, so as to always elicit the desired haptic sensation.

operative configurations, $i v)$ reduce as much as possible the dimension, encumbrance, and weight of the device. We present the mechanical analysis and theory behind this procedure, together with a MATLAB script to easily calculate the personalized dimensions. Finally, we also prepared an online tool for the Computer-Aided Design (CAD) software OnShape. Inputting the dimensions of the target fingertip and the target performance characteristics, the tool automatically adjusts the design of the wearable device and generates an STL file ready to be 3D printed.

\section{DEVICE DESCRIPTION}

We demonstrate our personalization technique for a representative 3-DoF wearable fingertip device, shown in Fig. 2.

The device is a parallel mechanism composed of a static upper body and a mobile platform (end-effector), connected by three articulated legs. The upper body and the motors are placed on the nail side of the finger, while the mobile endeffector is placed in contact with the finger pulp. The upper body and the end-effector are connected by three legs, each composed of two rigid links connected to each other, the body, and the end-effector according to a RRS kinematic chain. The revolute joints close to the upper body are actuated by servo motors. In each leg, the axes of the two revolute joints are parallel, so that it constitutes a 2-DoF planar articulated mechanism, constraining the motion of the center of each spherical joint on a plane fixed w.r.t. the body. Therefore, the mobile platform has 3 DoF w.r.t. the body. We use Hitech HS5035hd motors and a MicroMaestro (Pololu) control board.

A preliminary version of this device, designed without taking into account any user's specific characteristic, has been presented in [6] and in https://youtu.be/g7w5ejPa0sI. With respect to that system, our design has been modified to be suitable for personalization, as detailed in the following. A detailed analysis of the device in terms of control and dynamics response is reported in [7]. 


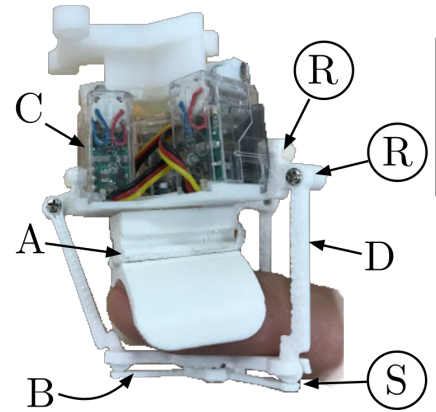

\begin{tabular}{|l|c|}
\hline Update Loop Time & $10 \mathrm{~ms}$ \\
Max. Roll Ang. $\psi_{M}$ & $30^{\circ}$ \\
Max. Pitch Ang. $\theta_{M}$ & $30^{\circ}$ \\
Max. Vert. Disp. $z_{M}$ & $10 \mathrm{~mm}$ \\
\hline
\end{tabular}

Fig. 2. The considered wearable haptic device. It is a $3-\mathrm{DoF}$ cutaneous module, composed of a static upper body (A) and a mobile platform (B): the body is located above the nail, supporting three servo motors (C), while the mobile platform contacts the finger pulp. Three legs (D) connect the mobile platform with the static body. Each leg is composed of two rigid links connected to each other and then with the body and the mobile platform, according to a RRS (Revolute-Revolute-Spherical) kinematic chain. Specifications and dimensions (w.r.t. Fig. 3) are reported on the right side.

\section{DEVICE ANALYSIS AND PERSONALIZATION}

\section{A. Problem introduction and main definitions}

In [6], [8], [7], we already summarized the main aspects of the device statics and kinematics model, following standard analysis procedures [9]. In this paper, we revise the modeling to motivate, parametrize, and evaluate the design choices of our device. The goal is to personalize the main geometrical characteristics of the device for a given fingertip and interaction. The criteria to take into account for choosing this optimized design are four-fold and listed in Sec. I. The scheme of the 3RRS parallel mechanism representing the wearable fingertip device is shown in Fig. 3 [10].

Let us indicate with $B_{i}, i=1,2,3$ the centers of the spherical joints on the mobile platform, and with $S_{1}=$ $\left\langle O_{1}, x_{1}, y_{1}, z_{1}\right\rangle$ the reference frame fixed on it, in which the origin $O_{1}$ and the axes are chosen as shown in Fig. 3. In each leg, the links are connected to each other through a revolute joint, whose axis is parallel to the one of the revolute joint fixed to the upper body. Let us indicate with $u_{i}$ the unit vector identifying, for each leg, the direction of the revolute joint axes. We can then define the plane $\pi_{i}$ passing through $B_{i}$ and perpendicular to $u_{i}$. The joint axes that intersect this plane in $A_{i}$ and $D_{i}$ correspond to the joint connecting each leg to the upper body and the middle joint, respectively. Let $S_{0}=\langle O, x, y, z\rangle$ be a reference frame on the upper body, in which the origin $O$ and the axes are chosen as shown in Fig. 3. Let us indicate with $a_{i}=\left[a_{i x}, a_{i y}, a_{i z}\right]^{\mathrm{T}}$ and $b_{i}=\left[b_{i x}, b_{i y}, b_{i z}\right]^{\mathrm{T}}$ the coordinates of $A_{i}$ and $B_{i}$, respectively, both expressed w.r.t. the $S_{0}$ frame, and with $b_{i}^{1}=\left[b_{i x}^{1}, b_{i y}^{1}, b_{i z}^{1}\right]^{\mathrm{T}}$ the coordinates of $B_{i}$ expressed w.r.t. the $S_{1}$. In general, the position and orientation of the mobile platform can be described by the vector $r=\left[r_{x}, r_{y}, r_{z}\right]^{\mathrm{T}}$ and $\operatorname{Roll}(\psi)-\operatorname{Pitch}(\theta)-\operatorname{Yaw}(\phi)$ angles $\varphi=[\psi, \theta, \phi]^{\mathrm{T}}$, respectively. As shown in [6], since the platform has 3 DoF, we can select three of these six variables and evaluate the remaining ones [7]. Let us indicate with $\xi$ a six-dimensional vector containing the mobile platform configuration (position and orientation): $\xi=\left[r^{\mathrm{T}}, \phi^{\mathrm{T}}\right]^{\mathrm{T}}$. Let us assume that the fingertip surface is known w.r.t. $S_{0}$, that it is convex and can be described by the function $z=f_{f}(x, y)$. For example, if the fingertip is

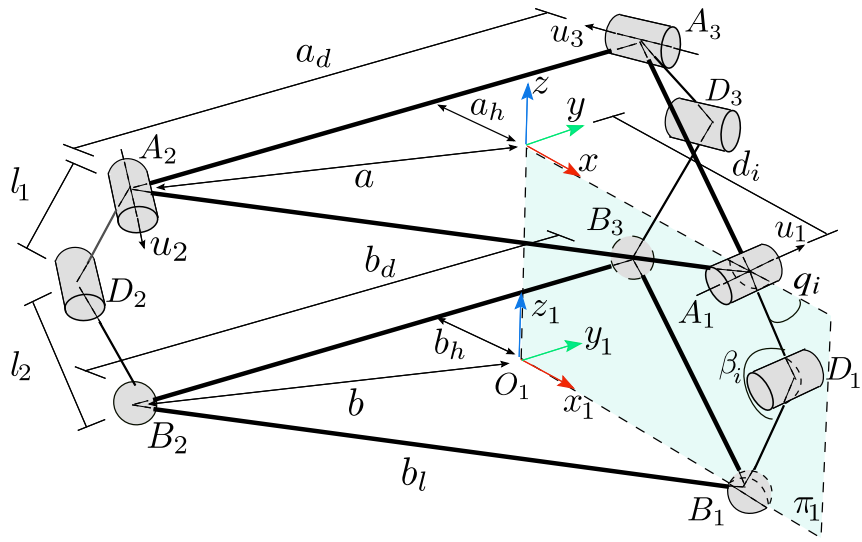

Fig. 3. Kinematic scheme of the 3-DoF cutaneous device. The structure of each leg is RRS (Revolute-Revolute-Spherical).

represented as a three-dimensional semi-ellipsoid with semiaxes $e_{1}, e_{2}$, and $e_{3}$, the corresponding representation is

$$
z=e_{3} \sqrt{1-\frac{x^{2}}{e_{1}^{2}}-\frac{y^{2}}{e_{2}^{2}}} .
$$

The device end-effector should be able to touch any point inside a given portion of the fingertip surface, indicated with $\mathscr{C}$. The dimension and shape of $\mathscr{C}$ can be evaluated on the basis of the target fingertip dimension, envisioned interaction, and device performance requirements, e.g., intended roll and pitch angles, vertical displacement.

\section{B. Constraints based on the user-specific features}

The device design is defined by three set of dimensions, that we seek to personalize: the dimensions of $a$ ) the mobile platform (end-effector), $b$ ) the static platform, and $c$ ) the articulated legs. The personalization process considers these three sub-problems in sequence:

- mobile platform (end-effector) dimensions are defined on the basis of the user's finger dimensions and the device target workspace, i.e., the surface of the finger that will be involved in the cutaneous stimulation.

- static platform dimensions are consequently defined so that, during the cutaneous stimuli application, only the mobile platform interacts with the fingertip and no undesired contacts with the legs occur.

- articulated legs lengths are defined to avoid kinematic singularities in any of the device operative configurations.

In the following, we provide a mathematical description of each step, constituting the basis for the automatic personalization procedure described in Sec. IV.

1) Preliminary constraints, symmetry: For fingertip devices, it is reasonable to assume that the device is symmetric with respect to the longitudinal plane (plane $x z$ ). Concerning the mobile platform, we can therefore assume $\overline{B_{1} B_{2}}=\overline{B_{1} B_{3}}=$ $b_{l}$ and $\overline{B_{2} B_{3}}=b_{d}$. The geometrical parameters $b_{d}, b$, and $b_{h}$, indicated in Fig. 3, define the shape of the mobile platform. In a generic configuration of the platform, indicating with $r$ the coordinates of $O_{1}$ w.r.t. $S_{0}$ and with $R$ the rotation matrix between $S_{1}$ and $S_{0}$, the coordinates of $B_{i}$ w.r.t. $S_{0}$ can be evaluated as $b_{i}=r+R b_{i}^{1}$. Since $B_{i}$ points move on the three fixed planes $\pi_{i}$ and according to the geometric dimensions 
indicated in Fig. 3, the following constraint equations hold

$$
b_{1, y}=0, \quad b_{2, x}=\frac{2 b_{h}}{b_{d}} b_{2, y}, \quad b_{3, x}=-\frac{2 b_{h}}{b_{d}} b_{3, y} .
$$

Eq. (2) introduces three constraints that limit the generic six-dimensional motion of the mobile platform to three DoF.

Moreover, since points $B_{i}$ have to move on fixed planes $\pi_{i}$, the following constraints on the upper static platform dimension hold $\frac{a_{d}}{a}=\frac{b_{d}}{b}=r_{p}$, where $r_{p}$ is an aspect ratio defining platform proportion between lateral and longitudinal direction. In particular, if $r_{p}=\sqrt{3}$, the platform has an equilateral structure; if $r_{p}<\sqrt{3}$, the platform is thinner in the lateral direction than in the longitudinal one.

2) Mobile platform dimension, contact with the fingertip: The dimensions of the mobile platform are evaluated on the basis of the first requirement reported in Sec. I, namely: i) reach all the parts of the fingertip needed for the given interaction. The mobile platform applies cutaneous stimuli to the fingertip. We call $\mathscr{C}$ the portion of the fingertip surface we want the mobile platform to reach. For simplicity, at this phase, we consider the theoretical single-point contact between the platform and the finger, i.e., we neglect the complex finger skin deformation caused by this interaction. For any configuration in which the platform is tangent to the fingertip in the generic point $P \in \mathscr{C}$, whose coordinates w.r.t. $S_{0}$ are $p=\left[p_{x}, p_{y}, p_{z}\right]^{T}, B_{i}$ have to belong to the plane tangent to the fingertip surface in $P$. For this reason, the coordinates of $B_{i}$ are related by three linear relationships:

$$
b_{i, z}=c_{1} b_{i, x}+c_{2} b_{i, y}+c_{3}
$$

where $i=1,2,3$, and $a, b, c$ are three coefficients that depend on the fingertip surface parameters and platform orientation:

$$
\begin{aligned}
c_{1} & =\left.\frac{\partial f_{f}}{\partial x}\right|_{p_{x}, p_{y}}, \quad c_{2}=\left.\frac{\partial f_{f}}{\partial y}\right|_{p_{x}, p_{y}} \\
c_{3} & =-\left.\frac{\partial f_{f}}{\partial x}\right|_{p_{x}, p_{y}} p_{x}-\left.\frac{\partial f_{f}}{\partial y}\right|_{p_{x}, p_{y}} p_{y}
\end{aligned}
$$

Three additional constraints are given by the distance between points $B_{i}$, which is constant

$$
\left(b_{i, x}-b_{j, x}\right)^{2}+\left(b_{i, y}-b_{j, y}\right)^{2}+\left(b_{i, z}-b_{j, z}\right)^{2}={\overline{B_{i} B_{j}}}^{2}
$$

with $i, j=1,2,3, i \neq j$. Eqs. (2), (3), (5) represent a $9 \times 9$ nonlinear system of equations whose solution gives the coordinates of the mobile platform vertexes $B_{i}$ for a given contact point $P$.

The device works properly if, for any point $P \in \mathscr{C}, P$ is also inside the triangle defined by $B_{i}$ points (i.e., it is reachable by the mobile platform). We can impose a constraint on the dimension of the mobile platform by checking that, for any platform configuration, point $P$ is inside the triangle defined by points $B_{1}, B_{2}$, and $B_{3}$. This constraint sets the lower bounds for the dimensions of the mobile platform. This constraint can only be indirectly verified: for a given fingertip shape and platform dimensions, we can only verify if the platform is able to reach every point of $\mathscr{C}$, so that the procedure has to be implemented iteratively. A simplified, yet approximated, solution can be obtained by choosing $b$ and $b_{d}$ so that the triangle that these parameters define is the minimum area triangle containing the $\mathscr{C}$ surface rectified on

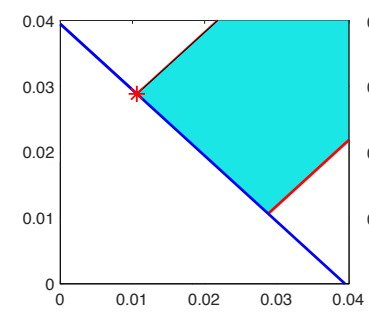

(a) $l_{2}$ vs. $l_{1}(\mathrm{~m})$ for

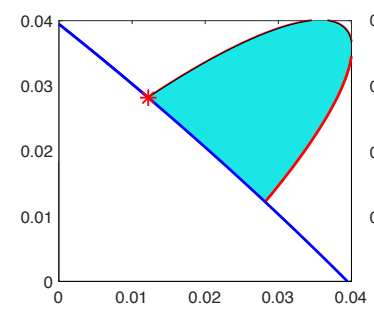

(c) $l_{2}$ vs. $l_{1}(\mathrm{~m})$ for

$\beta_{\text {min }}=0.15 \pi, \beta_{\text {max }}=0.85 \pi$ $\beta_{\text {min }}=0.01 \pi, \beta_{\max }=0.99 \pi$

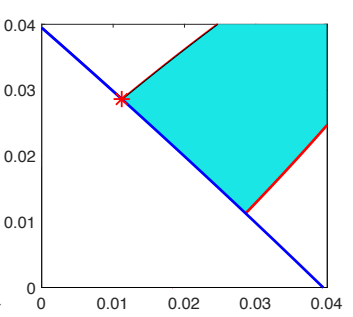

(b) $l_{2}$ vs. $l_{1}(\mathrm{~m})$ for

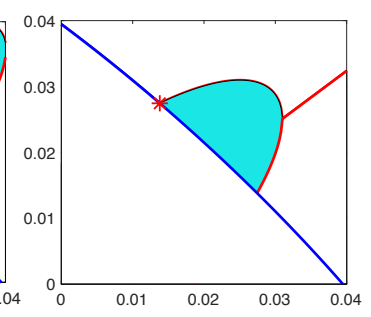

(d) $l_{2}$ vs. $l_{1}(\mathrm{~m})$ for $\beta_{\min }=0.1 \pi, \beta_{\max }=0.9 \pi$

Fig. 4. Choice of $l_{1}$ and $l_{2}$. Filled areas represent possible values of $l_{1}$ and $l_{2}$ considering different $\beta_{\min }$ and $\beta_{\max }$. Red curves represent equation $\beta=\beta_{\min }$, blue curves represent equation $\beta=\beta_{\max }$. The red star marker in case (a) represents the $l_{1}$ and $l_{2}$ values used for the prototype shown in Fig. 2.

the $x y$ plane. This simplified solution has been implemented in the MATLAB script submitted as supplemental material.

3) Static platform dimension, contact with finger lateral surface, aspect ratio: Once the mobile platform is defined, the dimensions of the static platform are evaluated to satisfy the second requirement introduced in Sec. I, specifically: ii) avoid undesired contacts between the device and the finger (e.g., lateral contacts). During haptic interaction, only the mobile platform should contact the fingertip, and any other contact between the articulated legs and the lateral surface of the fingertip should be avoided. To guarantee this behavior, we must ensure that, in any configuration of the platform, the lines connecting $A_{i}$ and $B_{i}$ do not intersect the fingertip surface. This constraint sets lower bounds for the dimensions of the static upper platform, which is defined by parameters $a$ and $a_{d}$ (see Fig. 3). However, if the dimension of the mobile platform, as defined in the previous Sec. III-B.2, is very small, this constraint can lead to quite a large static platform. To obtain a better proportion between the two platforms, we can set an additional constraint on the device aspect ratio, defined as $r_{d}=a / b$, imposing that it must fall within a fixed range, i.e., $r_{d, \min }<r_{d}<r_{d, \max }$.

4) Articulated leg dimensions: The dimensions of the articulated legs are evaluated to satisfy the third requirement in Sec. I, specifically: iii) avoid kinematic singularities in all the operative configurations. A configuration of the mobile platform can be defined by a vector $r$ and a rotation matrix $R$, corresponding to the contact with a generic point $P \in \mathscr{C}$. In any of these configurations, the device should be sufficiently far from singularities.

For any platform configuration $\xi$, the inverse kinematics procedure allows to evaluate corresponding rotations $q=$ $\left[q_{1}, q_{2}, q_{3}\right]^{\mathrm{T}}$ of the revolute joints in $A_{i}$ [6]. In other terms, the objective of the inverse kinematics is the definition of a function $f_{I K}: \mathbb{R}^{3} \rightarrow \mathbb{R}^{3}$ that allows to evaluate $q=f_{I K}(\xi)$. 
In particular, if the coordinates of $B_{i}$ w.r.t. $S_{0}$ are known, the actuator rotation angles $q_{i}$ can be evaluated as $q_{i}=\pi-\left(\alpha_{i}+\right.$ $\left.\delta_{i}\right)$, where

$\alpha_{i}=\arctan \left(\frac{s_{i z}}{\sqrt{s_{i x}^{2}+s_{i y}^{2}}}\right), \quad \delta_{i}=\arccos \left(\frac{l_{1}^{2}+s_{i}^{2}-l_{2}^{2}}{2 l_{1}\left|s_{i}\right|}\right)$,

with $s_{i}=b_{i}-a_{i}$, and $l_{1}=\left|\overline{D_{i} A_{i}}\right|$ and $l_{2}=\left|\overline{B_{i} D_{i}}\right|$ are the lengths of the proximal and distal link of each leg, respectively. The solution of the inverse kinematics problem is widely exploited in parallel mechanism analysis, and it is at the basis of our device position control [8]. In this paper, we use the results of the inverse kinematics to get insight for the design of the device dimensional parameters.

To avoid singular configurations during the motion, the "knee" angle $\beta_{i}$ has to ideally be comprised between 0 and $\pi$. Specifically, values of $\beta_{i}$ exactly equal to 0 or $\pi$ correspond to singular configurations of the mechanism, while values close to such limits lead to configurations in which motors are required to apply high torques, degrading the device performance. The criteria introduced in the previous Sections allow to define the dimensions of the mobile and static platforms, i.e., parameters $a, a_{d}, b, b_{d}$. Angles $\beta_{i}$ depend on these values and on the mechanism configuration, in particular on the magnitude of $s_{i}$ and on lengths $l_{1}$ and $l_{2}$. This new constraint $\beta_{\text {min }}<\beta_{i}<\beta_{\text {max }}$ leads to a system of non-linear inequalities whose solution provides indications on how to define $l_{1}$ and $l_{2}$. For example, considering $a, a_{d}, a_{h}, b, b_{d}$, and $b_{h}$ as reported in Fig. 2, filled areas in Fig. 4 show the subspace of $\left(l_{1}, l_{2}\right)$ pairs satisfying the constraints for different values of $\beta_{\min }$ and $\beta_{\max }$. Simply avoiding singular configurations does not provide a unique solution for $l_{1}$ and $l_{2}$, but rather a subspace of possible values. Among all the possible values of $\left(l_{1}, l_{2}\right)$ satisfying the constraints, we selected a solution that limits the overall device size and guarantees a suitable level of wearability and comfort, so as to satisfy the fourth requirement in Sec. I, namely: iv) reduce as much as possible the dimension, encumbrance, and weight of the device. In particular, we chose to keep $l_{1}$ as small as possible, so as to minimize motor torques for a given force to convey, as indicated with a red star in Fig. 4. Finally, since this criterion could lead to small $l_{1}$ values, which are difficult to manufacture, we considered an adjustable lower bound $l_{1, \min }$. The red star marker in Fig. 4 (a), located at $l_{1}=11 \mathrm{~mm}, l_{2}=28 \mathrm{~mm}$, indicates the design parameters of the device summarized in Fig. 2.

\section{DESIGN AND ANALYSIS PROCEDURE}

All the above-described criteria can be implemented in a numerical procedure that, given the user's finger characteristics and some hardware/manufacturing constraints, allows to dimension the device. We provide a MATLAB implementation of this procedure as a supplemental material. Moreover, a video, available as supplemental material and at https://youtu.be/FdTRPPIUJi8, summarizes the procedure.

One needs to provide the finger dimensions $e_{1}, e_{2}, e_{3}$ (see Fig. 5), the maximum roll angle $\psi_{\max }$, the maximum pitch angle $\theta_{\max }$, the maximum vertical displacement $z_{\max }$, and

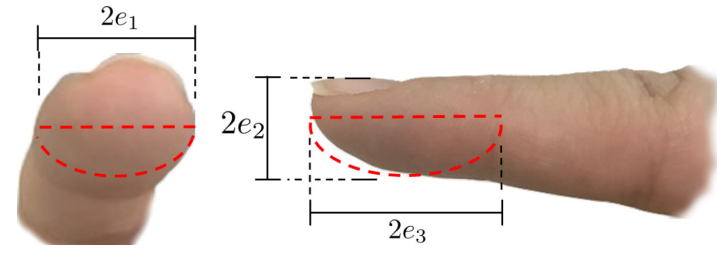

Fig. 5. The numerical procedure to optimize the device design uses these fingertip geometric characteristics as inputs.

a fixed offset $h$ between the finger and the static platform. This last input can be considered to model the thickness of the static platform as well as the presence of a layer of soft material reducing the pressure on the top of the finger (e.g., foam). In the script, for the sake of simplicity, platform aspect ratio $r_{p}$ has been considered constant and the device ratio $r_{d}$ has not been constrained as described in Sec. III-B.3. Nonetheless, both aspects can be easily considered as further design/input variables in the optimization procedure.

The procedure for defining the optimal device dimensions is divided in three main parts: the first one sets the dimension of the mobile platform (see Sec. III-B.2), the second part defines the dimensions of the static platform (see Sec. IIIB.3), and the third part allows to define the optimal length of the device legs (see Sec. III-B.4). The outputs of this procedure are the optimized dimensions of the device, i.e., $a$, $a_{d}, b, b_{d}, l_{1}, l_{2}$. Examples of outputs for four representative fingertips are shown in Tab. I. Fig. 6 shows, for the same four fingertips, the contact patch $\mathscr{C}$ rectified on the $x y$ plane and the optimized mobile platform as evaluated by our procedure. The filled areas in Fig. 6 represent, as in Fig. 4 , the set of $\left(l_{1}, l_{2}\right)$ pairs that avoid kinematic singularities in all the operative configurations of the device. The red stars represent the $\left(l_{1}, l_{2}\right)$ pair chosen by the automatic personalization procedure. As we can see, in all cases but $7 \mathrm{a}$, the procedure chooses the pair minimizing $l_{1}$, so as to reduce as much as possible the torque required from the actuators. In case $7 \mathrm{a}$, following this approach would provide an $l_{1}$ too small $\left(l_{1}<l_{\text {min }}\right)$, so the procedure settles for $l_{1}=l_{\text {min }}$. Fig. 8 shows, for the same four representative fingertips, a front view ( $y z$ plane) of the finger wearing its personalized device. The distance between the fixed platform and the finger was $h=8 \mathrm{~mm}$ for all the configurations. It is interesting to notice that the procedure does not provide a mere scaling of the device, but instead a more complex personalization and optimization of the device structure and kinematics.

For each representative finger, we also calculated the ratio between the device and finger volumes, $r_{v}$. The former is approximated as the volume of the polyhedron defined by points $A_{i}, B_{i}$ and $D_{i}$, while the latter is approximated as the union between a semi-ellipsoid with semi-axes $e_{1}, e_{2}$, and $e_{3}$ and a cylinder with an elliptical base with semi-axes $e_{1}$ and $e_{2}$ and height $e_{3}$ (in red in Fig. 5). As expected, this ratio varies in the different configurations: it is higher for small fingers, due to the fixed constraints that were imposed (e.g., $l_{1 \text { min }}$ and $h$ ), and it tends to decrease as the dimension of the finger increases. Finally, we also calculated the same device/fingertip volume ratio, $r_{v, n o}$, assuming that all the fingers wear the same device, i.e., the largest one (so that it fits everyone). In this 


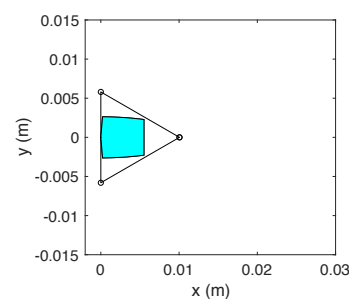

(a) top view (m)

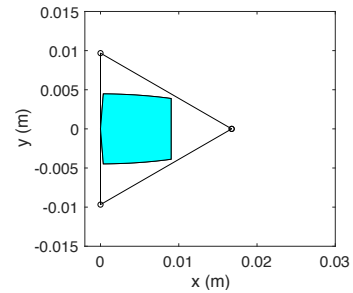

(c) top view (m)

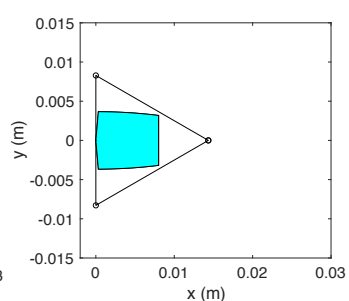

(b) top view (m)

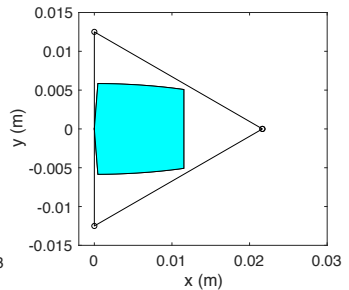

(d) top view (m)
Fig. 6. Rectified contact patch $\mathscr{C}$ and mobile platform profile for the four representative fingertips and configurations (a), (b), (c), (d) of Tab. I.

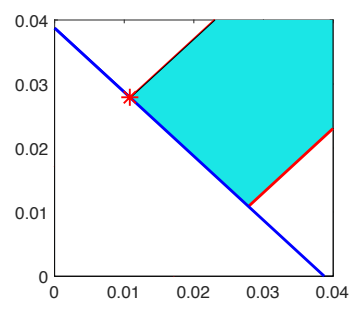

(a) $l_{2}$ vs. $l_{1}(\mathrm{~m})$

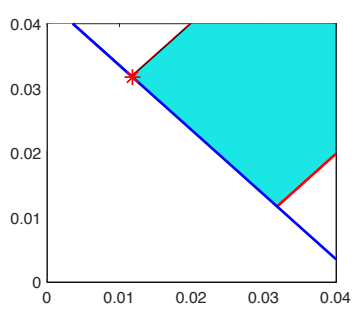

(c) $l_{2}$ vs. $l_{1}(\mathrm{~m})$

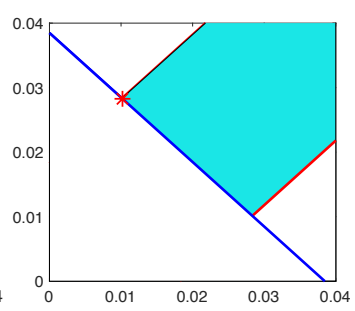

(b) $l_{2}$ vs. $l_{1}(\mathrm{~m})$

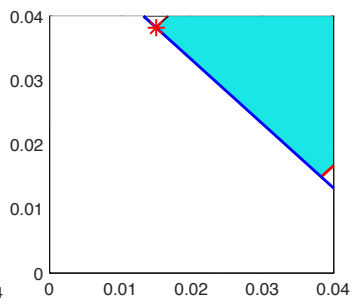

(d) $l_{2}$ vs. $l_{1}(\mathrm{~m})$
Fig. 7. Choice of $l_{1}$ and $l_{2}$ avoiding singular configurations for each of the four representative fingertips and configurations (a), (b), (c), (d) of Tab. I. Filled areas represent possible pairs of $l_{1}$ and $l_{2}$ avoiding singular configurations, similarly to diagrams reported in Fig. 4. The red star marker shows the choices made by the automatic customization procedure.

case the ratio has a much wider variability. This analysis shows the importance of our personalization. If we design a platform that is too small, we cannot reach all the fingertip surface; conversely, if we design a platform that is too big, we ask the user to wear an unnecessarily bulky device.

Finally, these parameters can be used in any CAD software to get the solid model of the optimized device. As an example, we have prepared a parametric CAD design on the online software OnShape. Starting from the optimized dimensions of the device as described above, the design automatically adjusts and generates a solid model ready to be 3D-printed. The resource is available at https://tinyurl.com/yx8ucceo. A user needs to register to OnShape (free of charge) and then copy the workspace so as to be able to edit it. Variables can be modified in the tab named "VARIABLES," while the adjusted design can be seen in the tab named "assembleddevice." As the user modifies the dimension variables in the

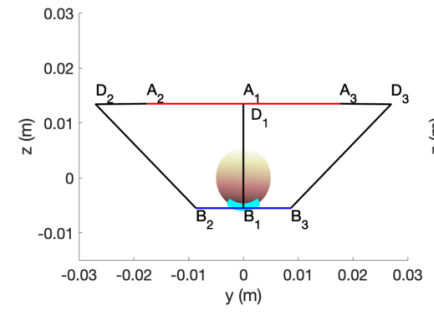

(a) front view (m)

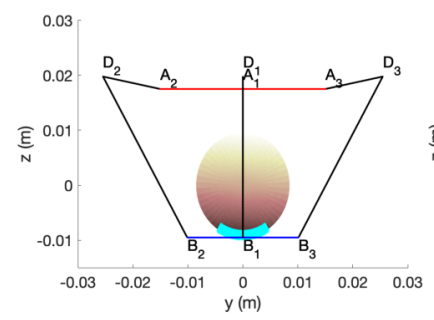

(c) front view $(\mathrm{m})$
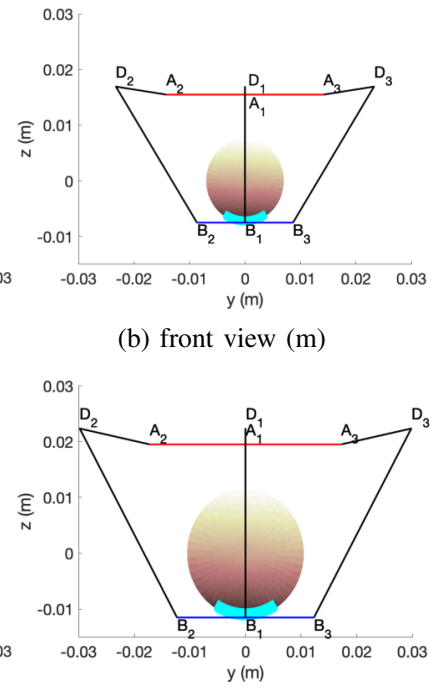

(d) front view (m) (b) front view (m)

Fig. 8. Front views of the finger (pink dashed ellipse) and thimble structure for the four representative fingertips and configurations (a), (b), (c), (d) of Tab. I. Red line represents the front view of the fixed platform, blue line the mobile platform, black lines the legs. Cyan dots represent the surface (projected on the $y z$ plane) that the device can reach. The device is represented in the reference configuration.

first tab, the design modifies accordingly. Finally, the design can be exported in different formats (e.g., STL) and realized using any additive manufacturing technology, as for instance a standard 3D printer. We have included the device STEP files as supplemental material.

\section{USE CASE}

We carried out a preliminary evaluation aimed at showing an effect in using a personalized vs. a general-purpose device.

The experimental setup is composed of a Leap Motion tracking system, one wearable fingertip device, and a virtual environment composed of a 3-dimensional sphere. Subjects are required to wear the fingertip device on the right index finger and interact with the virtual environment. The subjects hand pose is tracked using the Leap Motion, and a virtual hand mimicked the hand pose in the virtual environment. Every time the index finger came in contact with the virtual sphere, the wearable device applied a suitable amount of force to the fingertip (similarly to what we did in [6], [11]). Users were able to see the virtual environment through a screen placed in front of them. The Leap Motion was placed on a table between the user and the screen. Seven participants ( 5 males, 2 females) took part to the experiment. The task consisted of interacting with the virtual environment for two 5-minutes periods: once wearing a general-purpose wearable device $(\mathrm{G})$, with the same dimensions described in Sec. II and Fig. 2, and once wearing a personalized device $(\mathrm{P})$ generated following our procedure, presented in a randomized order. Users could interact with the sphere as they liked, e.g., poking, throwing, squeezing, or juggling it.

We evaluated the experience through 4 Likert-type questions, asking users to rate the "coherence of the haptic feedback with respect to the virtual environment (Q1)," the "quality of the haptic feedback received (Q2)," the "wearability of the system (Q3)," and "the comfort of the system (Q4)" in 
TABLE I

EXAMPLE OF DEVICE CHARACTERISTICS AS EVALUATED BY OUR PROCEDURE (DIMENSIONS IN $\mathrm{mm}$. VALUES IN BOLD ARE PROVIDED BY THE USER. THE OTHER INPUTS ARE SET TO $\psi_{\max }=\theta_{\max }=30^{\circ}, z_{\max }=10 \mathrm{~mm}, h=8 \mathrm{~mm}, l_{1 \min }=8 \mathrm{~mm}$.

\begin{tabular}{|c||c|c|c||c|c|c|c|c|c|c|c|c|}
\hline Ex. & $e_{1}$ & $e_{2}$ & $e_{3}$ & $a$ & $a_{d}$ & $b$ & $b_{d}$ & $l_{1}$ & $l_{2}$ & $r_{v}$ & $r_{v, n o}$ & Figs. \\
\hline$(a)$ & $\mathbf{5 . 5}$ & $\mathbf{5}$ & $\mathbf{1 1}$ & 20 & 35 & 10 & 17 & 11 & 28 & 7.08 & 16.68 & $6 \mathrm{a}, 7 \mathrm{a}, 8 \mathrm{a}$ \\
$(b)$ & $\mathbf{7 . 5}$ & $\mathbf{7}$ & $\mathbf{1 6}$ & 16 & 28 & 10 & 17 & 11 & 29 & 2.27 & 6.00 & $6 \mathrm{~b}, 7 \mathrm{~b}, 8 \mathrm{~b}$ \\
$(c)$ & $\mathbf{9 . 5}$ & $\mathbf{8 . 5}$ & $\mathbf{1 8}$ & 17 & 30 & 11 & 20 & 12 & 34 & 1.88 & 3.46 & $6 \mathrm{c}, 7 \mathrm{c}, 8 \mathrm{c}$ \\
$(d)$ & $\mathbf{1 3 . 5}$ & $\mathbf{1 1}$ & $\mathbf{2 3}$ & 21 & 37 & 16 & 27 & 16 & 44 & 1.47 & 1.47 & $6 \mathrm{~d}, 7 \mathrm{~d}, 8 \mathrm{~d}$ \\
\hline
\end{tabular}

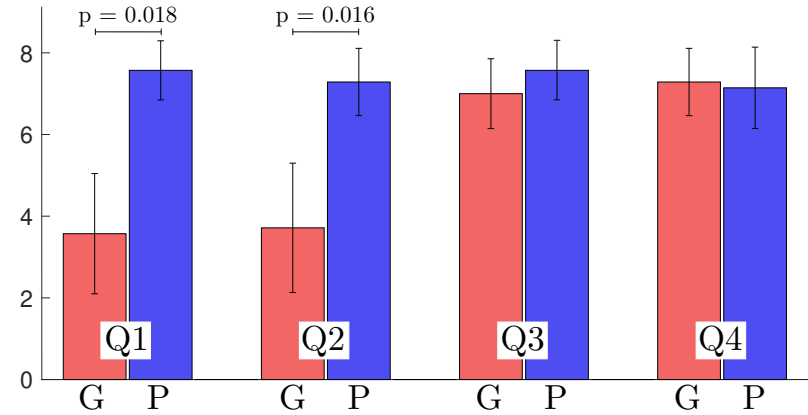

Fig. 9. Virtual Reality (VR) interaction: results. Mean and $95 \%$ confidence interval of the answers to the questionnaire when using the general-purpose device $(\mathrm{G})$ vs. the personalized device $(\mathrm{P})$. Higher is better.

the two conditions (general-purpose vs. personalized device, $\mathrm{G}$ vs. P). Each assertion is rated with a score from 1 ("completely disagree" with the assertion) to 9 ("completely agree"). To determine whether the answers for the conditions differed, we ran one Wilcoxon signed-rank tests $(\mathrm{a}=0.05)$ per question, see Fig. 9. The personalized device showed significantly increased performance regarding the coherence (Q1) and quality (Q2) of the interaction, while no significant difference was registered for the wearability $(\mathrm{Q} 3)$ and comfort $(\mathrm{Q} 4)$. The latter is probably due to the fact that the personalized devices were, depending on the user, sometimes larger and sometimes smaller than the general-purpose one.

\section{DisCUSSION AND CONCLUSION}

We presented a novel approach for personalizing a wearable haptic device for a target fingertip, taking into account its specific geometrical characteristics as well as some target performance metrics.

The procedure tackles four sub-tasks sequentially: symmetry and aspect ratio, customization of the mobile platform (i.e., definition of $b$ and $b_{d}$ parameters), customization of the static platform, (i.e., definition of $a$ and $a_{d}$ parameters), and definition of the articulated leg lengths $l_{1}$ and $l_{2}$. This approach does not consider the process as a global optimization problem and, for this reason, it could lead to suboptimal results - but it also presents some advantages. First, the proposed process can be directly managed by the user, who can easily add or remove constraints and features without significantly impacting the overall algorithm structure. For instance, if the user decides to produce different devices with the same static platform (e.g., to speed up the manufacturing process), the proposed personalization procedure can be easily modified to act on the mobile platform and articulated legs only. Second, since each sub-task has been solved in an analytic way, its software implementation is straightforward, does not need any specific optimization tool, and can also be integrated in parametric CAD systems. Together with the mechanical analysis and evaluation of the adapted design, we present an automatic procedure to calculate the personalized device dimensions as well as an online CAD utility for generating a ready-to-3D-print STL file of the adapted design. Any user can design a personalized version of our device using this technique.

Our analysis can be completed by assessing the device kinematics, quasi-static modeling, and manipulability, extending the formulation presented in [6]. Of course, it is always important to know that a device should consider its target application. Toward this objective, from our parametric analysis, researchers can further adjust the design to their specific needs, to achieve different target performance and wearability levels according to the field of application. Another point to highlight is that, of course, the shape of a fingertip is not exactly that of an ellipsoid, and discrepancies between our ellipsoidal model and the actual fingertip might result in suboptimal choices for the design.

In the future, we will focus on the evaluation of this method, analyzing in depth the difference in performance and comfort between using a general-purpose device and one personalized through our procedure.

\section{REFERENCES}

[1] C. Pacchierotti, S. Sinclair, M. Solazzi, A. Frisoli, V. Hayward, and D. Prattichizzo, "Wearable haptic systems for the fingertip and the hand: Taxonomy, review, and perspectives," IEEE Trans. Haptics, vol. 10, no. 4, pp. 580-600, 2017.

[2] K. Dandekar, B. I. Raju, and M. A. Srinivasan, "3-D finite-element models of human and monkey fingertips to investigate the mechanics of tactile sense," J. Biom. Eng., vol. 125, no. 5, pp. 682-691, 2003.

[3] A. Courtney and M. Ng, "Hong Kong female hand dimensions and machine guarding," Ergonomics, vol. 27, no. 2, pp. 187-193, 1984.

[4] C. Pacchierotti, D. Prattichizzo, and K. J. Kuchenbecker, "Displaying sensed tactile cues with a fingertip haptic device," IEEE Trans. Haptics, vol. 8, no. 4, pp. 384-396, 2015.

[5] E. Young, D. Gueorguiev, K. J. Kuchenbecker, and C. Pacchierotti, "Compensating for fingertip size to render tactile cues more accurately," IEEE Trans. Haptics, 2020.

[6] F. Chinello, C. Pacchierotti, M. Malvezzi, and D. Prattichizzo, "A three revolute-revolute-spherical wearable fingertip cutaneous device for stiffness rendering," IEEE Trans. Haptics, vol. 11, pp. 39-50, 2017.

[7] F. Chinello, M. Malvezzi, D. Prattichizzo, and C. Pacchierotti, "A modular wearable finger interface for cutaneous and kinesthetic interaction: control and evaluation," IEEE Trans. Industrial Electronics, vol. 67, no. 1, 2020.

[8] F. Chinello, M. Malvezzi, C. Pacchierotti, and D. Prattichizzo, "Design and development of a 3rrs wearable fingertip cutaneous device," in Proc. IEEE/ASME Intl. Conf. on Advanced Intelligent Mechatronics, 2015, pp. 293-298.

[9] L.-W. Tsai, Robot analysis: the mechanics of serial and parallel manipulators. John Wiley \& Sons, 1999.

[10] J. Li, J. Wang, W. Chou, Y. Zhang, T. Wang, and Q. Zhang, "Inverse kinematics and dynamics of the 3-rrs parallel platform," in Proc. IEEE Intl. Conf. Robotics and Automation, vol. 3, 2001, pp. 2506-2511.

[11] L. Meli, S. Scheggi, C. Pacchierotti, and D. Prattichizzo, "Wearable haptics and hand tracking via an rgb-d camera for immersive tactile experiences," in Proc. ACM SIGGRAPH Posters, 2014. 